\title{
Sensitivity analysis for the Euler equations in Lagrangian coordinates
}

\author{
Christophe Chalons, Régis Duvigneau and Camilla Fiorini
}

\begin{abstract}
Sensitivity analysis (SA) is the study of how changes in the inputs of a model affect the outputs. SA has many applications, among which uncertainty quantification, quick evaluation of close solutions, and optimization. Standard SA techniques for PDEs, such as the continuous sensitivity equation method, call for the differentiation of the state variable. However, if the governing equations are hyperbolic PDEs, the state can be discontinuous and this generates Dirac delta functions in the sensitivity. The aim of this work is to define and approximate numerically a system of sensitivity equations which is valid also when the state is discontinuous: to do that, one can define a correction term to be added to the sensitivity equations starting from the Rankine-Hugoniot conditions, which govern the state across a shock. We show how this procedure can be applied to the Euler barotropic system with different finite volumes methods.
\end{abstract}

Key words: Sensitivity analysis, $p-$ system, Dirac delta function, Finite Volume.

MSC (2010): 35L60, 65M08, 49Q12.

\section{Introduction}

Sensitivity analysis (SA) concerns the quantification of changes in Partial Differential Equations (PDEs) solution due to perturbations in the model input. It has been a

Christophe Chalons and Camilla Fiorini

Laboratoire de Mathématiques de Versailles, UVSQ, CNRS, Université Paris-Saclay,

45 avenue des États-Unis, 78035 Versailles, France

e-mail: christophe.chalons@uvsq.fr, camilla.fiorini@uvsq. fr

Régis Duvigneau

Université Côte d'Azur, INRIA, CNRS, LJAD, INRIA Sophia-Antipolis Méditerranée Center, ACUMES Project-Team, 2004 route des Lucioles - B.P. 93, 06902 Sophia Antipolis, France

e-mail: regis.duvigneaulinria.fr 
topic of active research for the last years, due to its many applications, for instance in uncertainty quantification, quick evaluation of close solutions [4], and optimization [2], to name but a few. Note that SA approaches differ from adjoint methods, which are restricted to the evaluation of functional derivatives. Standard SA methods work only under certain hypotheses of regularity of the solution $\mathbf{U}$ [1]. These assumptions are not verified in the case of hyperbolic systems of the general form

$$
\left\{\begin{array}{l}
\partial_{t} \mathbf{U}+\partial_{x} \mathbf{F}(\mathbf{U})=0, \quad x \in \mathbb{R}, \quad t>0, \\
\mathbf{U}(x, 0)=\mathbf{U}_{0}(x),
\end{array}\right.
$$

due to possible discontinuities, which can occur even when the initial condition is smooth. If the state $\mathbf{U}$ is discontinuous, Dirac delta functions will appear in the sensitivity $\mathbf{U}_{a}=\partial_{a} \mathbf{U}$. Here and throughout this work, $a$ denotes the input parameter of the model which may vary and induce a non trivial sensitivity $\mathbf{U}_{a}$ of the state solution $\mathbf{U}$.

In this work, we consider the Euler equations in Lagrangian coordinates in a barotropic case, i.e. the $p$-system:

$$
\left\{\begin{array}{l}
\partial_{t} \tau-\partial_{x} u=0, \\
\partial_{t} u+\partial_{x} p(\tau)=0,
\end{array}\right.
$$

where $\tau>0$ is the covolume (i.e. $\tau=\frac{1}{\rho}$, where $\rho$ is the density of the fluid), $u$ is the velocity and the pressure $p(\tau)$ is a function of $\tau$ such that $p^{\prime}(\tau)<0$ and $p^{\prime \prime}(\tau)>0$. Under these assumptions, (1) is strictly hyperbolic with eigenvalues $\lambda_{ \pm}= \pm c$ where $c=\sqrt{-p^{\prime}(\tau)}$ is the Lagrangian sound speed. In this work we will consider $p(\tau)=$ $\tau^{-\gamma}$, where $\gamma=1.4$ is the heat capacity ratio.

By differentiating the system (1) with respect to the parameter of interest $a$ and considering smooth solutions of (1), we obtain the sensitivity equations:

$$
\left\{\begin{array}{l}
\partial_{t} \tau_{a}-\partial_{x} u_{a}=0 \\
\partial_{t} u_{a}+\partial_{x}\left(p^{\prime}(\tau) \tau_{a}\right)=0
\end{array}\right.
$$

One can define the following vectors:

$$
\mathbf{U}=\left[\begin{array}{l}
\tau \\
u
\end{array}\right], \quad \mathbf{F}(\mathbf{U})=\left[\begin{array}{c}
-u \\
p(\tau)
\end{array}\right], \quad \mathbf{U}_{a}=\left[\begin{array}{c}
\tau_{a} \\
u_{a}
\end{array}\right], \quad \mathbf{F}_{a}\left(\mathbf{U}, \mathbf{U}_{a}\right)=\left[\begin{array}{c}
-u_{a} \\
p^{\prime}(\tau) \tau_{a}
\end{array}\right],
$$

and rewrite the systems (1) and (2) in a vectorial form:

$$
\left\{\begin{array}{l}
\partial_{t} \mathbf{U}+\partial_{x} \mathbf{F}(\mathbf{U})=0, \\
\partial_{t} \mathbf{U}_{a}+\partial_{x} \mathbf{F}_{a}\left(\mathbf{U}, \mathbf{U}_{a}\right)=0 .
\end{array}\right.
$$

At this stage, it is easy but important to remark that the global system (3) admits the same real eigenvalues $\lambda_{ \pm}$(both with multiplicity 2 ) as the original system (1) but it is only weakly hyperbolic as soon as $\tau_{a} \neq 0$. We recall that weak hyperbol- 
icity means that the Jacobian matrix of the system admits real eigenvalues but is not $\mathbb{R}$-diagonalizable. As a consequence and without any modification of (3), discontinuous weak solutions of the state variable $\mathbf{U}$ will generally induce Dirac delta functions in the sensitivity variable $\mathbf{U}_{a}$, in addition to the usual discontinuity, so that the solutions of (3) have to be understood in the sense of measures. However, and as already stated above, we are not interested in considering Dirac delta functions. Instead, we would like to introduce a modification in the sensitivity equations in order to make the system (3) valid in the usual sense of weak solutions also for discontinuous state variables (as done in [5]). This is the aim of the next section.

\section{Source term}

In order to remove the Dirac delta functions that are naturally present in the solutions of (3), we suggest to add to (2) a source term $\mathbf{S}$, which is of the following form:

$$
\mathbf{S}=\sum_{k=1}^{N_{s}} \delta_{k} \boldsymbol{\rho}_{k}
$$

where $N_{s}$ is to be associated to the number of discontinuities in the state solution $\mathbf{U}$, $\boldsymbol{\rho}_{k}$ is the amplitude of the $k$-th correction (to be computed), and $\delta_{k}$ is the Dirac delta function $\delta_{k}=\delta\left(x-x_{s, k}\right)$, where $x_{s, k}$ is the position of the $k$-th discontinuity. Let us consider then a control volume $\left(x_{1}, x_{2}\right) \times\left(t_{1}, t_{2}\right)$ containing a single discontinuity indexed by $k$ and propagating at speed $\sigma_{k}$. By integrating the equations (2) with the additional source term (4) over the control volume, when the size of the control volume tends to zero one has:

$$
\boldsymbol{\rho}_{k}(t)=\left(\mathbf{U}_{a}^{-}-\mathbf{U}_{a}^{+}\right) \sigma_{k}+\mathbf{F}_{a}^{+}-\mathbf{F}_{a}^{-},
$$

where the plus (respectively minus) indicates the value of the sensitivity $\mathbf{U}_{a}$ and of the flux $\mathbf{F}_{a}$ to the right (respectively left) of the discontinuity. In other words, (5) gives a natural meaning of $\boldsymbol{\rho}_{k}$ in terms of a defect measure of the Rankine-Hugoniot relations applied to (2). It is now a matter of defining $\boldsymbol{\rho}_{k}$ in such a way that the new model including the source term is also valid for discontinuities of the state variable (recall that (2) was obtained by differentiating with respect to $a$ the smooth solutions of (1)). Considering the Rankine-Hugoniot conditions across a discontinuity of the state variable, namely $\left(\mathbf{U}^{-}-\mathbf{U}^{+}\right) \sigma_{k}=\mathbf{F}^{-}-\mathbf{F}^{+}$, we differentiate with respect to the parameter $a$ to obtain $\left(\mathbf{U}_{a}^{-}-\mathbf{U}_{a}^{+}\right) \sigma_{k}+\left(\mathbf{U}^{-}-\mathbf{U}^{+}\right) \sigma_{k, a}=\mathbf{F}_{a}^{-}-\mathbf{F}_{a}^{+}$, with $\sigma_{k, a}=\partial_{a} \sigma_{k}$. Comparing the latter with (5), one is thus led to set

$$
\boldsymbol{\rho}_{k}(t)=\sigma_{k, a}\left(\mathbf{U}^{+}-\mathbf{U}^{-}\right) .
$$




\section{Numerical schemes}

We introduce a constant space step $\Delta x$ and a varying time step $\Delta t^{n}$. We define the mesh interfaces $x_{j+1 / 2}=j \Delta x$, the cells $C_{j}=\left[x_{j-1 / 2}, x_{j+1 / 2}\right]$, the cell centres $x_{j}$ and the intermediate times $t^{n+1}=t^{n}+\Delta t^{n}$, where $\Delta t^{n}$ is chosen according to the usual CFL condition.

\section{The Godunov method.}

In this paragraph, we present the usual Godunov method based on the exact resolution of the Riemann problem including the source term, and associated with the initial data $\mathbf{U}(x, 0)=\mathbf{U}_{L} \mathbb{1}_{\left(x<x_{c}\right)}+\mathbf{U}_{R} \mathbb{1}_{\left(x>x_{c}\right)}$ and $\mathbf{U}_{a}(x, 0)=\mathbf{U}_{a, L} \mathbb{1}_{\left(x<x_{c}\right)}+\mathbf{U}_{a, R} \mathbb{1}_{\left(x>x_{c}\right)}$. The details are not reported here but one has been able to prove that the analytical solution is known and that its structure is resumed in Figures 1-2. In particular, the solution for the state consists of two waves, which can be either shocks or rarefaction waves, and whose speed can be computed exactly. On the other hand, the sensitivity has the same two-wave structure, however both of the waves are discontinuities. This simplification for the sensitivity is due to the fact that we are considering a reduced Euler system, under barotropic conditions (cf. [6]).

Since the state equations (1) are conservative, the Godunov method can be written with the classic update formula

$$
\mathbf{U}_{j}^{n+1}=\mathbf{U}_{j}^{n}-\frac{\Delta t}{\Delta x}\left(\mathbf{F}\left(\mathbf{U}_{j+1 / 2}^{*}\right)-\mathbf{F}\left(\mathbf{U}_{j-1 / 2}^{*}\right)\right),
$$

where $\mathbf{U}_{j-1 / 2}^{*}$ denotes the exact intermediate state variable in the Riemann solution associated to left and right states in the $j-1$-th and $j$-th cells.

Due to the presence of the source term in the sensitivity equations, a conservative update formula like (7) cannot be obtained. However, recall that the structure of the sensitivity at each interface is very simple and made of two shocks. As a consequence, one can easily perform the average on the cells, provided that the slopes $\kappa_{1}$ and $\kappa_{2}$, i.e. the slope of the red lines and the blue solid line in Figure 2, are known at each interface $j-1 / 2$. More precisely, we easily get

$$
\mathbf{U}_{a, j}^{n+1}=\mathbf{U}_{a, j}^{n}+\frac{\Delta t}{\Delta x}\left(\kappa_{2, j-1 / 2}\left(\mathbf{U}_{a, j-1 / 2}^{*}-\mathbf{U}_{a, j}^{n}\right)-\kappa_{1, j+1 / 2}\left(\mathbf{U}_{a, j+1 / 2}^{*}-\mathbf{U}_{a, j}^{n}\right)\right)
$$

where the intermediate states $\mathbf{U}_{a, j-1 / 2}^{*}$ and $\mathbf{U}_{a, j+1 / 2}^{*}$ are known analytically and depend on $\mathbf{U}_{j-1 / 2}^{*}$ and $\mathbf{U}_{j+1 / 2}^{*}$ respectively. We remark that $\kappa_{\ell, j \pm 1 / 2}$ depends on the solution structure: in case of shock it is given by the Rankine-Hugoniot conditions, in case of rarefaction wave it is the eigenvalue $\lambda$ evaluated in the intermediate state $\mathbf{U}_{j \pm 1 / 2}^{*}$. Let us observe that (8) already encompasses the source term to remove the Dirac delta function, therefore we do not need to discretise it. However, this correction is taken into account even in a numerical rarefaction profile (we recall that two points in a rarefaction wave are not necessarily linked by a rarefaction), and this is the cause of the failure of Godunov's method. In the next section, we present a Roe's method which uses shock detectors in order to overcome this problem. 


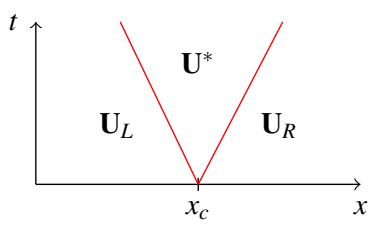

(a) 1-shock-2-shock.

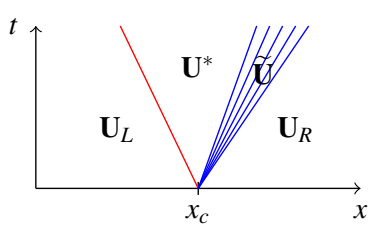

(b) 1-shock-2-rarefaction

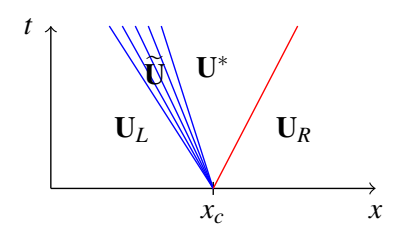

(c) 1-rarefaction-2-shock.

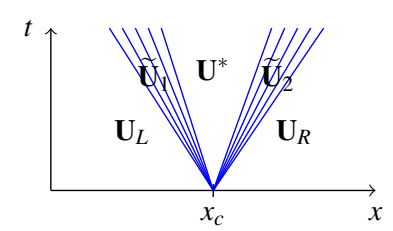

(d) 1-rarefaction-2-rarefaction.

Fig. 1: Configurations for the state variable $\mathbf{U}$.

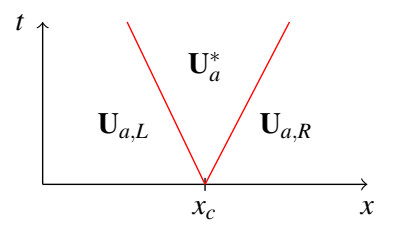

(a) 1-shock-2-shock.

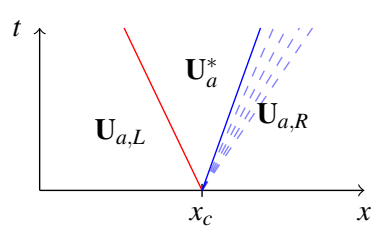

(b) 1-shock-2-shock.

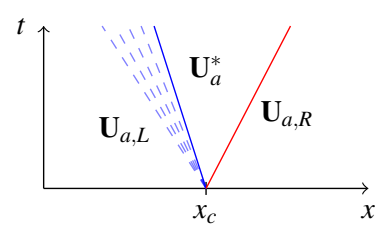

(c) 1-shock-2-shock.

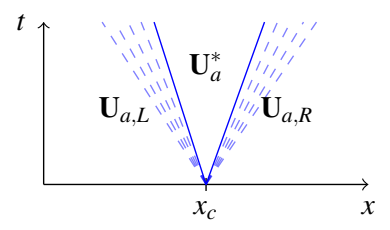

(d) 1-shock-2-shock.

Fig. 2: Corresponding configurations for the sensitivity $\mathbf{U}_{a}$.

\section{A Roe-type method.}

The proposed approximate Riemann solver of Roe-type consists of three constant states (say $\mathbf{U}_{L}, \mathbf{U}^{*}$ and $\mathbf{U}_{R}$ for the state, and $\mathbf{U}_{a, L}, \mathbf{U}_{a}^{*}$ and $\mathbf{U}_{a, R}$ for the sensitivity) separated by two shock waves propagating at velocities $\lambda_{L, j-1 / 2}^{R O E}=$ $-\sqrt{-\left(p\left(\tau_{j-1}^{n}\right)-p\left(\tau_{j}^{n}\right)\right) /\left(\tau_{j-1}^{n}-\tau_{j}^{n}\right)}$ and $\lambda_{R, j-1 / 2}^{R O E}=-\lambda_{L, j-1 / 2}^{R O E}$ if $\tau_{j-1}^{n} \neq \tau_{j}^{n}$ (and of course $\mp \sqrt{-p^{\prime}\left(\tau_{j}^{n}\right)}$ otherwise). In the following, we will use the notation $\lambda_{j-1 / 2}^{R O E}=\lambda_{R, j-1 / 2}^{R O E}=-\lambda_{L, j-1 / 2}^{R O E}$. The fact that the velocities at each interface are equal and opposite in sign allows us to write at the interface $j-1 / 2$ the Harten, Lax and van Leer consistency relations for the state in the following way: 


$$
\mathbf{U}_{j-1 / 2}^{*}=\frac{1}{2}\left(\mathbf{U}_{j-1}^{n}+\mathbf{U}_{j}^{n}\right)-\frac{\mathbf{F}\left(\mathbf{U}_{j}^{n}\right)-\mathbf{F}\left(\mathbf{U}_{j-1}^{n}\right)}{2 \lambda_{j-1 / 2}^{R O E}} .
$$

Knowing $\mathbf{U}_{j-1 / 2}^{*}$ and the velocity $\lambda_{j-1 / 2}^{R O E}$ at each interface one can average the solution value on the cells, obtaining the following update formula for the state:

$$
\mathbf{U}_{j}^{n+1}=\mathbf{U}_{j}^{n}-\frac{\Delta t}{\Delta x}\left(\Phi_{j+1 / 2}^{n}-\Phi_{j-1 / 2}^{n}\right),
$$

where $\Phi^{n}$ is the numerical flux and is defined as follows:

$$
\Phi_{j-1 / 2}^{n}=\frac{\mathbf{F}\left(\mathbf{U}_{j}^{n}\right)+\mathbf{F}\left(\mathbf{U}_{j-1}^{n}\right)}{2}-\frac{\lambda_{j-1 / 2}^{R O E}}{2}\left(\mathbf{U}_{j}^{n}-\mathbf{U}_{j-1}^{n}\right) .
$$

We now consider the sensitivity equation, with the following source term, defined according to equations (4) to (6) and given by

$$
\Delta x \mathbf{S}_{j-1 / 2}=-\partial_{a} \lambda_{j-1 / 2}^{R O E}\left(\mathbf{U}_{j-1 / 2}^{*}-\mathbf{U}_{j-1}^{n}\right) d_{1, j-1}+\partial_{a} \lambda_{j-1 / 2}^{R O E}\left(\mathbf{U}_{j}^{n}-\mathbf{U}_{j-1 / 2}^{*}\right) d_{2, j},
$$

where $d_{\ell, j}$ is a shock detector to be defined, and $d_{\ell, j}=1$ if there is an $\ell$-shock in the $j$-th cell, it is zero elsewhere. The shock detector used in this work is based on the fact that the velocity $u$ is always decreasing across a shock, and the covolume $\tau$ is decreasing across a 1 -shock, whilst it is increasing across a 2 -shock.

Finally, the update formula for the sensitivity is the following:

$$
\mathbf{U}_{a, j}^{n+1}=\mathbf{U}_{a, j}^{n}-\frac{\Delta t}{\Delta x}\left(\Phi_{a, j+1 / 2}^{n}-\Phi_{a, j-1 / 2}^{n}\right)+\frac{\Delta t}{2}\left(\mathbf{S}_{j-1 / 2}+\mathbf{S}_{j+1 / 2}\right),
$$

where $\Phi_{a}^{n}$ is defined as follows:

$$
\Phi_{a, j-1 / 2}^{n}=\frac{\mathbf{F}_{a}\left(\mathbf{U}_{j}^{n}, \mathbf{U}_{a, j}^{n}\right)+\mathbf{F}_{a}\left(\mathbf{U}_{j-1}^{n}, \mathbf{U}_{a, j-1}^{n}\right)}{2}-\frac{\lambda_{j-1 / 2}^{R O E}}{2}\left(\mathbf{U}_{a, j}^{n}-\mathbf{U}_{a, j-1}^{n}\right) .
$$

We remark that in (11), we add the source term $\mathbf{S}_{j-1 / 2}$ to both cells $j-$ th and $(j-$ 1)-th: indeed, it is defined starting from an integral balance done on both cells.

Finally, we extended this scheme to the second order: we used a standard twostep Runge-Kutta method in time, whereas in space we used a MUSCL scheme adapted to take into account a second order discretization of the source term.

\section{Numerical results}

In this section we present some numerical results. The spatial domain is $(0,1), x_{c}=$ 0.5 , and final time $T=0.03$.

The test case considered is a 1-shock-2-rarefaction, with the following initial conditions: $\mathbf{U}_{L}=(0.7,0)^{T}, \mathbf{U}_{R}=(0.2,0)^{T}, \mathbf{U}_{a, L}=(0,1)^{T}, \mathbf{U}_{a, R}=(0,0)^{T}$, and the 
parameter of interest is $a=u_{L}$. Figure 3 shows the state $\tau$ and its sensitivity $\tau_{a}$ ( $u$
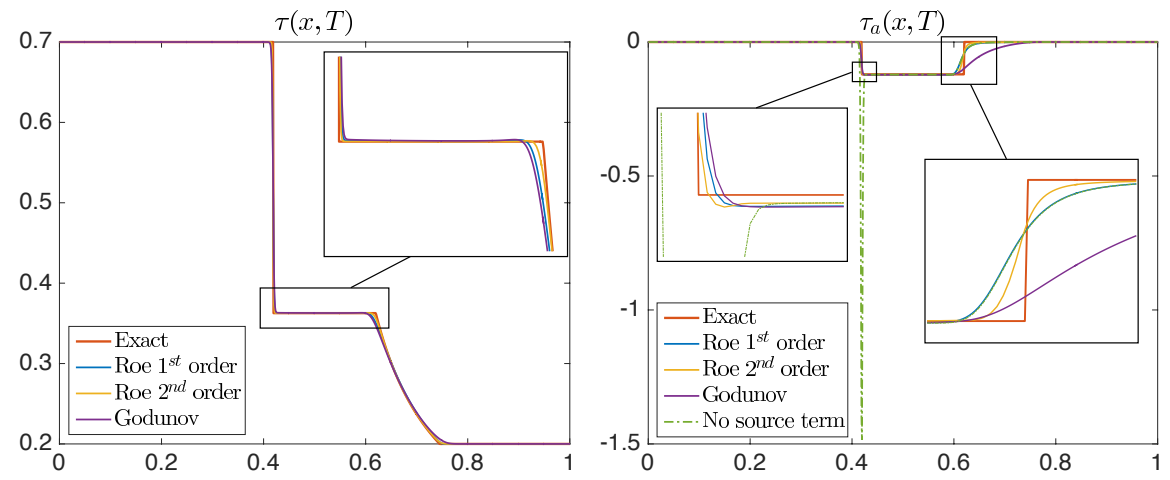

Fig. 3: Test case: 1-shock - 2-rarefaction, $\Delta x=10^{-3}$

and $u_{a}$ have a similar behaviour) at the final time $T$. As one can see, all the methods approximate well the state solution and the modified sensitivity formulation succeeds in removing the Dirac delta function located at point $x \approx 0.4$ and noticeable without source term (scheme labelled "No source term" in Figure 3), however we remark that there are two main problems in the sensitivity solution: the shock corresponding to the state rarefaction is not captured and the value in the star zone is not correct. The first problem can be solved by refining the mesh or by using higher-order schemes (one can observe that the second order Roe method captures the discontinuity better); whilst the second problem, in our opinion, is due to the numerical diffusion in the shock. Figure 4 shows the convergence of the schemes: for the state we have the expected convergence; concerning the sensitivity, for coarser meshes the error is decreasing because its main part is in the rarefaction zone, however when this contribution becomes comparable with the error in the star zone, a plateau is reached.

\section{Conclusion and discussion}

The numerical results show that the modified sensitivity system here proposed is well defined and it allows us to achieve the main goal of this work, i.e. to have a sensitivity without Dirac delta function. However, the proposed modified formulation yields an incorrect value of the sensitivity in the star zone. Interestingly, numerical results show that even the exact Godunov method does not provide a correct solution, neither does a higher order scheme. In our opinion, this problem is due to the numerical diffusion in the shock. To illustrate this, we briefly show in Figure 5 the results obtained with a modified Godunov method based on sampling techniques, 

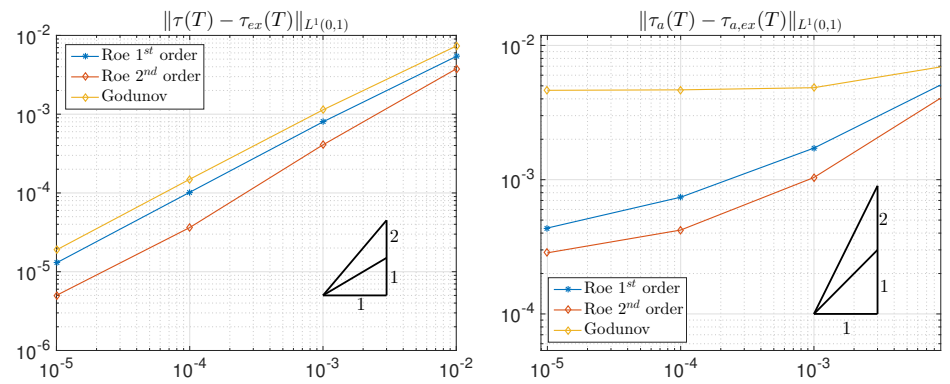

Fig. 4: Convergence results for test case 1-shock - 2-rarefaction.
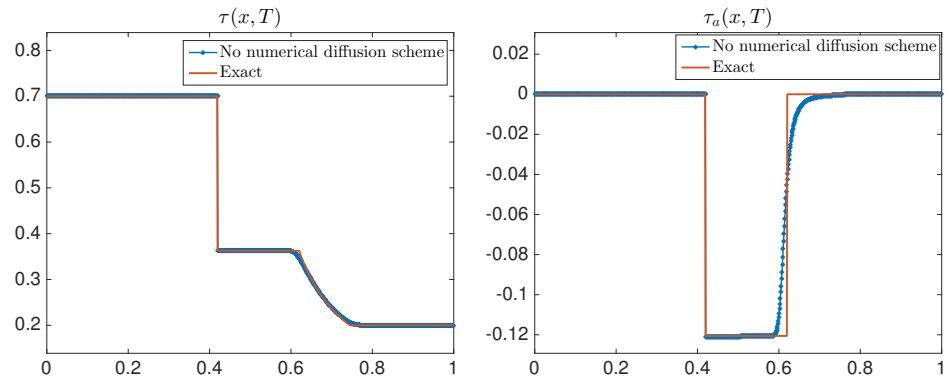

Fig. 5: Scheme with no numerical diffusion in the shock.

introduced in [3], which does not have any numerical diffusion and seems to provide a correct sensitivity in the star region.

\section{References}

1. C. Bardos and O. Pironneau. A formalism for the differentiation of conservation laws. Compte rendu de l'Académie des Sciences, 335(10):839-845, 2002.

2. J. Borggaard and J. Burns. A PDE sensitivity equation method for optimal aerodynamic design. Journal of Computational Physics, 136(2):366 - 384, 1997.

3. C. Chalons and P. Goatin. Godunov scheme and sampling technique for computing phase transitions in traffic flow modeling. Interfaces and Free Boundaries, 10(2):197-221, 2008.

4. R. Duvigneau and D. Pelletier. A sensitivity equation method for fast evaluation of nearby flows and uncertainty analysis for shape parameters. Int. J. of CFD, 20(7):497-512, August 2006.

5. V. Guinot. Upwind finite volume solution of sensitivity equations for hyperbolic systems of conservation laws with discontinuous solutions. Computers \& Fluids, 38(9):1697-1709, 2009.

6. V. Guinot, M. Leménager, and B. Cappelaere. Sensitivity equations for hyperbolic conservation law-based flow models. Advances in water resources, 30(9):1943-1961, 2007. 